\title{
Helical structure of the waves propagating in a spinning Timoshenko beam
}

\author{
By K. T. Chan $^{1}$, N. G. Stephen ${ }^{2}$ and S. R. Reid ${ }^{3}$ \\ ${ }^{1}$ Department of Mechanical Engineering, The Hong Kong Polytechnic \\ University, Hung Hom, Kowloon, HKSAR, People's Republic of China \\ (mmktchan@polyu.edu.hk) \\ ${ }^{2}$ Mechanical Engineering, School of Engineering Sciences, The University of \\ Southampton, Highfield, Southampton SO17 1BJ, UK \\ ${ }^{3}$ School of Mechanical, Aerospace and Civil Engineering, The University of \\ Manchester, PO Box 88, Sackville Street, Manchester M60 1QD, UK
}

The aim of the paper is to study the cause of a frequency-splitting phenomenon that occurs in a spinning Timoshenko beam. The associated changes in the structure of the progressive waves are investigated to shed light on the relationship between the wave motion in a spinning beam and the whirling of a shaft. The main result is that travelling bending waves in a beam spinning about its central axis have the topological structure of a revolving helix traced by the centroidal axis with right-handed or left-handed chirality. Each beam element behaves like a gyroscopic disc in precession being rotated at the wave frequency with anticlockwise or clockwise helicity. The gyroscopic effect is identified as the cause of the frequency splitting and is shown to induce a coupling between two interacting travelling waves lying in mutually orthogonal planes. Two revolving waves travelling in the same direction in space appear, one at a higher and one at a lower frequency compared with the pre-split frequency value. With reference to a given spinning speed, taken as clockwise, the higher one revolves clockwise and the lower one has anticlockwise helicity, each wave being represented by a characteristic four-component vector wavefunction.

Two factors are identified as important, the shear-deformation factor $q$ and the gyroscopic-coupling phase factor $\theta$. The $q$-factor is related to the wavenumber and the geometric shape of the helical wave. The $\theta$-factor is related to the wave helicity and has two values, $+\pi / 2$ and $-\pi / 2$ corresponding to the anticlockwise and clockwise helicity, respectively. The frequency-splitting phenomenon is addressed by analogy with other physical phenomena such as the Jeffcott whirling shaft and the property of the local energy equality of a travelling wave. The relationship between Euler's formula and the present result relating to the helical properties of the waves is also explored.

Keywords: helical wave; propagating wave; four-component vector wavefunction; gyroscopic effect; Timoshenko beam; Euler's formula

\section{Introduction}

A normal mode of vibration of a spinning beam will be described as the superposition of a revolving $\mathrm{s}_{\mathrm{a}}$-standing and $\mathrm{s}_{\mathrm{b}}$-standing wave (Chan et al. 2002) 
in the beam. Although the present study is focussed on the travelling wave, a review of the more practical, standing wave structure will be given first, since a standing wave is a combination of two oppositely travelling identical waves.

Previous studies (Bishop 1959; Bishop \& Parkinson 1968; Morton \& Johnson 1980; Morton 1985) were restricted to using Euler-Bernoulli beam (EB) theory and were mainly concerned with synchronous vibration due to unbalanced masses. More recently, these have been supplemented with studies using Timoshenko beam (TB) theory on vibration, leading to the present study of wave motion in spinning shafts.

Using EB theory, Bauer (1980) identified Coriolis inertia effects as the cause of the doubling of the number of natural frequencies of a spinning beam. Lee et al. (1988) used Rayleigh beam (RB) theory and then Zu \& Han (1992) used TB theory to show that the gyroscopic effect is important.

However, their theoretical studies are phenomenological, being focused only on modal descriptions of shaft vibration. Their results did not show the interesting helical properties of the waves that constitute the normal modes they predicted using the standard method for finding modal solutions. The study of wave phenomena in a spinning beam is important because the influence of the gyroscopic effect on the waves underlies the frequency-splitting phenomenon as studied by many previous investigators.

Argento \& Scott (1995), Kang \& Tan (1998) and Tan \& Kang (1998) sought flexural wave solutions for a spinning Timoshenko beam. They were able to describe the reflection, transmission and dispersion characteristics of the waves in the spinning beam. However, their results concerning gyroscopic precession are not very clear. One should distinguish between the precession of the spinning beam per se and the precession of a wave propagating in it. From the present study, it is shown that the precession of the wave is free motion that manifests as a corkscrew-like helix traced by the centroidal axis of the spinning beam, which revolves about the axis of the beam at rest to transfer the kinetic and potential energy with the wave, forward or backward along the beam. For clarity, revolving or precession is used for the rotation about the axis of the beam at rest to differentiate it from rotation of a cross-sectional beam element about a transverse axis passing through the centroid in the plane of cross-section. For a helical bending shape, a neutral plane does not exist and so one should be cautious in using the usual definition of a neutral axis, see $\$ 2$. The above studies also failed to show the helical properties and the gyroscopic-precession motion of bending waves travelling in the spinning beam. This leaves the behaviour and structure of the travelling waves in the spinning beam and their relation to the observed behaviour of the shafts to be explored.

In order to address the latter, a new approach is used that is related to the wave-mechanics approach employed by Chan et al. (2002). Using this approach, one may describe the phenomena in terms of their constituent waves, their dispersion behaviour and their relationship to the normal modes. However, the wave-mechanics approach to the spinning beam problem is quite detailed and is worthy of a separate treatment in its own right, which the first author is undertaking. The approach used here does not rely on this new treatment, and is based on the concept of superposed standing waves in a Timoshenko beam (Chan et al. 2002). The solution obtained by using this provides a description of the physical phenomena in terms of the geometrical structure of the wave 
shape, the evolution of the topology of the wave in time and space along the beam axis, the force and moment distributions and energy distributions that cause the changes. All these descriptions are directly related to the dispersion and propagation properties of the waves.

The following picture of wave phenomena in a spinning beam emerges. A uniform beam with circular cross-section spins at $\Omega \mathrm{rad} \mathrm{s}^{-1}$ about the fixed $z$-axis that coincides with the undeformed centroidal axis of the beam at rest. A travelling wave will cause the straight centroidal axis of the beam to deform and trace the shape of a helix revolving at $\Omega \mathrm{rad} \mathrm{s}^{-1}$ about the $z$-axis. The helix has a constant radius $W_{\mathrm{o}}$ and a pitch. The revolving motion of the helix is regarded as the integrated motion of the precession of each of the crosssectional elements spinning at $\Omega$ about the helically shaped centroidal axis, called the centroidal helix. If the beam is divided into many individual cross-sectional elements, each of them will spin about an axis normal to the cross-section. The angle between the axis normal to the cross-section and the tangent to the centroidal helix at that location is the shear deformation. The shape of the centroidal helix is maintained by the internal stress field due to bending and shear and the inertia associated with the revolving motion of the wave.

The revolving motion of the corkscrew-like centroidal helix described earlier will be shown to conform to the energy-transfer process of a progressive wave. This is intimately related to the application of a local property of a travelling wave that the local kinetic and potential energy are equal. Comparing the present energy equality result with those of Elmore \& Heald (1985) will show some subtle differences as well as similarities.

With the present model, the whirling of a shaft can be viewed as the generation of two types of revolving standing waves in superposition, a revolving $\mathrm{s}_{\mathrm{a}}$-standing and $\mathrm{s}_{\mathrm{b}}$-standing wave. Each type has a right-handed $(\mathrm{RH})$ and a lefthanded (LH) helical wave component, both subject to a synchronous excitation, clockwise or anticlockwise. By this model, the studies of Bishop \& Parkinson (1968) and Morton (1985) for EB beams can be extended to a spinning Timoshenko beam. However, this will not be considered further herein.

\section{Theory of helical waves in a Timoshenko beam}

\section{(a) Various rotational motions with respect to the inertial-coordinate system}

A fixed right-handed $x y z$-coordinate system is used with the $z$-axis coinciding with the centroidal axis of the Timoshenko beam at rest. All the bending waves (waves for short) travel along the $z$-axis only. The beam spins about the $z$-axis when there is no wave travelling. However, the centroidal axis is deformed into a helix (centroidal helix) if a wave travels along the $z$-axis. The helix represents the shape of the travelling wave, revolving about the $z$-axis. A number of rotational motions of the beam are defined here for purpose of clarity. The rotational motion of a beam element about the axis normal to its crosssection at the centroid is referred to as its spin, and its speed $\Omega$ is called its spinning speed. It is clear that the local direction of spin varies along the centroidal helix. The rotation of a wave is referred to as the rotation of the polarization of the wave. It presents as a revolving centroidal helix as shown in figures 1 and 2. The revolving motion of the helix can be described as the 


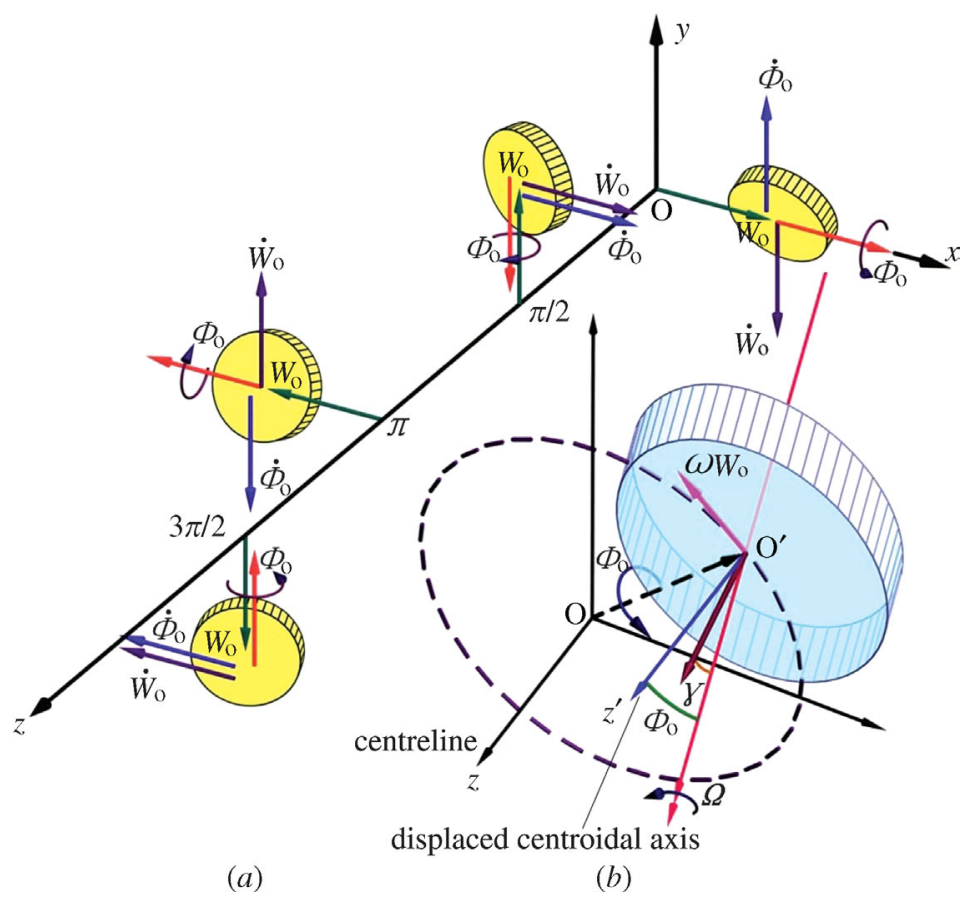

Figure 1. (a) Distributed gyroscopic elements of the spinning beam induced by a progressive bending wave. (b) Gyroscopic precession of a spinning beam element (enlarged size).

gyroscopic precession of each of the spinning beam elements as illustrated in figure $1 b$. The rotation (in radians) of a beam cross-section $\phi$ about a transverse axis is referred to as bending rotation. Its amplitude is $\Phi_{\mathrm{o}}$ and direction as shown in the same figure. In the figure, $\mathrm{O}^{\prime} z^{\prime}$ is parallel to the $\mathrm{O} z$ axis.

\section{(b) Definitions of helicity and chirality of bending waves}

The helical properties of the wave will be deduced by solving the equation of motion of a spinning Timoshenko beam. The cross-section of the beam is assumed uniform along the axis but initially the shape is taken as asymmetric. Although the result of the present study can be applied to beam cross-section with rotational symmetry about its centroidal axis, for purposes of brevity, we will use the circular cross-section. When the cross-section is circular, an important factor $\theta$ of $\mathrm{e}^{\mathrm{i} \theta}$ is identified as two-valued and equal to either positive or negative $\pi / 2$, being associated with the revolving directions of the wave concerned, written as the four-component vector wavefunction

$$
\boldsymbol{s}=\left\{\begin{array}{c}
w_{x} \\
\phi_{y} \\
w_{y} \\
\phi_{x}
\end{array}\right\}=\left\{\begin{array}{c}
W_{x} \\
\pm \mathrm{i} \Phi_{y} \\
\mathrm{e}^{\mathrm{i} \theta} W_{y} \\
\pm \mathrm{i} \mathrm{e}^{\mathrm{i} \theta} \Phi_{x}
\end{array}\right\} \mathrm{e}^{\mathrm{i}(\omega t \pm k z)}
$$



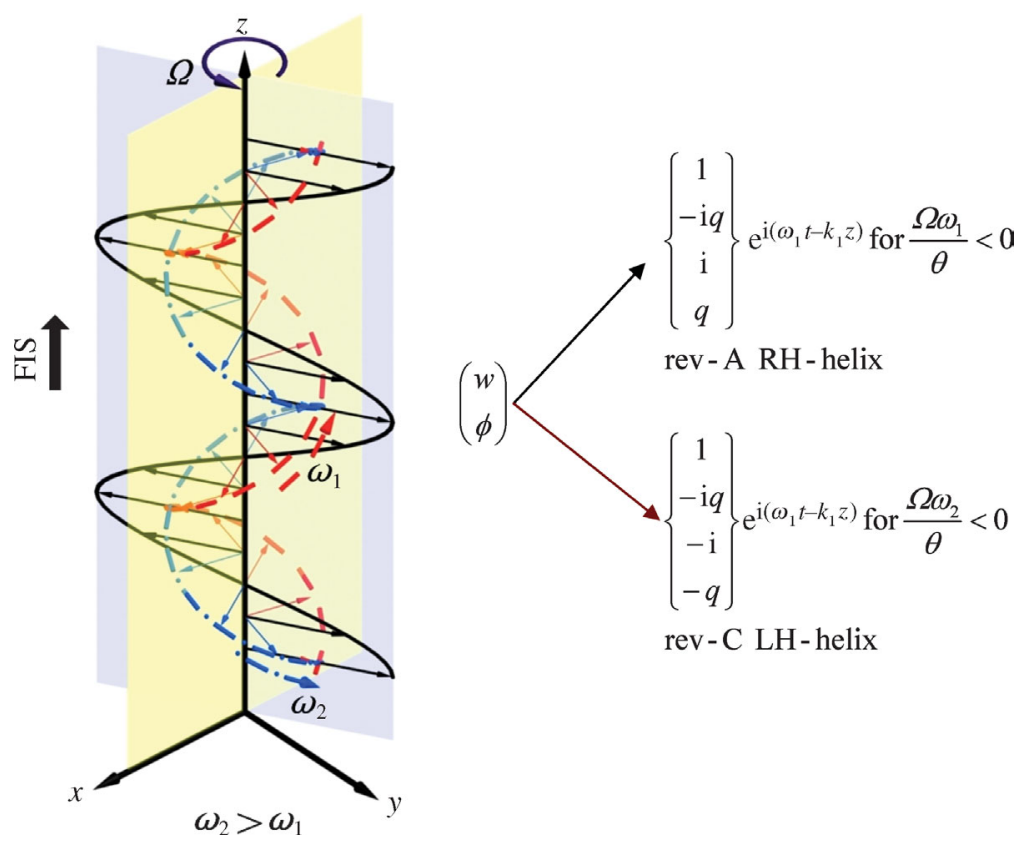

rev-C LH-helix

Figure 2. Splitting of a plane-polarized traveling sinusoidal FIS wave (solid line) into a rev-A RH-helix FIS wave (dash line) and a rev-C LH-helix FIS wave (dash-dot line) arising from the frequency-splitting induced by the gyroscopic effect. The rev-A wave revolves at $\omega_{1}$ and rev-C wave revolves at $\omega_{2}$, where $\omega_{2}>\omega_{1}$. The plane-polarized wave is not revolving before splitting. The curves in this figure are traced by the centroidal axis.

where the ' + ' and ' - ' signs are for the backward-in-space (BIS) waves and forward-in-space (FIS), respectively. Upper case symbols $W_{x}, W_{y}, \Phi_{x}$ and $\Phi_{y}$ represent the corresponding amplitudes, $\omega$ and $k$ are the angular frequency and wavenumber of the bending wave motion along the beam axis, respectively, $\omega$ being positive definite.

For a travelling wave with frequency $\omega$, the wavenumber $k$ equals $k_{x}$ or $k_{y}$ the subscript being used to indicate the direction of the transverse displacement of the wave in the $x$ - or $y$-axis, respectively. However, $k_{x}$ and $k_{y}$ are equal when the beam is circular. In equation $(2.1), \mathrm{e}^{\mathrm{i} \theta}$ is taken as a factor to define an interacting relation between the two waves in the mutually orthogonal planes. It will be shown that such interaction manifests as a coupling arising from the gyroscopic effect.

Equation (2.1) represents either an anticlockwise wave or a clockwise wave, depending on the sign of $\theta$. Given either value of $\theta$, the bending wave assumes the shape of a perfect helix, traced by the centroidal axis of the beam, revolving at $\omega$ and the orbit of each of the centroidal points is circular. One may refer to $\mathrm{e}^{\mathrm{i} \theta}= \pm \mathrm{i}$ as the helicity of a revolving wave. Thus, an anticlockwise wave has helicity $\mathrm{e}^{\mathrm{i} \pi / 2}=+\mathrm{i}$ and a clockwise wave has helicity $\mathrm{e}^{-\mathrm{i} \pi / 2}=-\mathrm{i}$. Given the helicity, the sign before the wavenumber $k$ in $\mathrm{e}^{\mathrm{i}(\omega t \pm k z+\theta)}$ determines the handedness of the centroidal helix, left-handed or right-handed corkscrew-like chirality. 


\section{(c) Equation of motion and four-component vector wave solution}

(i) Beam properties, coordinate system and equations

While the main results will pertain to a circular cross-section beam, to start from an asymmetric cross-section first helps to bring out the $\theta$-factor that can be identified as the value for characterizing a beam. This transpires to be a simple factor in this case, but is otherwise time dependent.

The nomenclatures of the beam properties are given first. The beam is assumed to be infinitely long. For an arbitrary cross-section, with respect to the fixed coordinate system in which the $z$-axis is along the beam axis, the product and second moments of areas about the $x$ - and $y$-axes, $I_{x y}, I_{x}$ and $I_{y}$, are not invariant with respect to time. Likewise neither are the shear coefficients $\kappa_{x}$ and $\kappa_{y}$ with respect to the centroidal translations in $x$ - and $y$-axes, respectively. The cross-sectional area, the density, the shear modulus of rigidity, and Young's modulus, $A, \rho, G$ and $E$, are taken as constant for the uniform and homogeneous beam. The spinning speed $\Omega$ is taken as positive according to the conventional right-hand screw rule.

Short-form notations for partial differential operators are used, for example, $\partial_{t}^{2}=\left(\partial^{2} / \partial t^{2}\right), \quad \partial_{z}^{2}=\left(\partial^{2} / \partial z^{2}\right), \partial_{t}=(\partial / \partial t), \partial_{z}=(\partial / \partial z)$, etc. The operator $\boldsymbol{D}$ for the bending wave propagating in the spinning Timoshenko beam is given as

$$
D=\left[\begin{array}{cccc}
\rho A \partial_{t}^{2}-\kappa_{x} G A \partial_{z}^{2} & \kappa_{x} G A \partial_{z} & 0 & 0 \\
-\kappa_{x} G A \partial_{z} & \rho I_{y} \partial_{t}^{2}-E I_{y} \partial_{z}^{2} & 0 & \Omega \rho\left(I_{x}+I_{y}\right) \partial_{t} \\
& +\kappa_{x} G A & & -\rho I_{x y} \partial_{t}^{2} \\
0 & 0 & \rho A \partial_{t}^{2}-\kappa_{y} G A \partial_{z}^{2} & \kappa_{y} G A \partial_{z} \\
0 & -\Omega \rho\left(I_{x}+I_{y}\right) \partial_{t} & -\kappa_{y} G A \partial_{z} & \rho I_{x} \partial_{t}^{2}-E I_{x} \partial_{z}^{2} \\
& -\rho I_{x y} \partial_{t}^{2} & & +\kappa_{y} G A
\end{array}\right]
$$

The equation of motion with respect to $\boldsymbol{D}$ operating on the $s$ of equation (2.1) is written as

$$
D s=0,
$$

where $\mathbf{0}=\left\{\begin{array}{llll}0 & 0 & 0 & 0\end{array}\right\}^{\mathrm{T}}$ for free motion of the waves in the beam. Equation (2.3) with operator $\boldsymbol{D}$ written as in equation (2.2) is for a general case of a uniform beam with asymmetric cross-section. It will become apparent that, when the cross-section of the uniform beam is circular, operator $\boldsymbol{D}$ is reduced to a skew-symmetric matrix so that the wavefunctions in equation (2.3) represents a travelling wave with a perfect helix shape traced by the centroidal axis revolving either clockwise or anticlockwise.

First, define the amplitude ratios

$$
q_{x}=\frac{\Phi_{y}}{W_{x}} \text { and } q_{y}=\frac{\Phi_{x}}{W_{y}} .
$$

By substitution, equation (2.1) becomes

$$
s=\left\{\begin{array}{c}
w_{x} \\
\phi_{y} \\
w_{y} \\
\phi_{x}
\end{array}\right\}=\left\{\begin{array}{c}
W_{x} \\
\pm \mathrm{i} q_{x} W_{x} \\
\mathrm{e}^{\mathrm{i} \theta} W_{y} \\
\pm \mathrm{i} \mathrm{e}^{\mathrm{i} \theta} q_{y} W_{y}
\end{array}\right\} \mathrm{e}^{\mathrm{i}(\omega t \pm k z)}
$$


Substituting equation (2.5) into (2.3), differentiating the wavefunction with respect to $z$ and $t$, and separating the real and imaginary parts, one can write four characteristic equations as

$$
\begin{gathered}
\rho\left(I_{x}+I_{y}\right) q_{y} \Omega \omega \cos \theta+\rho I_{x y} q_{y} \omega^{2} \sin \theta=0 \\
\rho\left(I_{x}+I_{y}\right) q_{x} \Omega \omega W_{x}+\left[\kappa_{y} G A k_{y}-q_{y}\left(E I_{x} k_{y}^{2}+\kappa_{y} G A-\rho I_{x} \omega^{2}\right)\right] W_{y} \sin \theta=0, \\
{\left[\rho\left(I_{x}+I_{y}\right) q_{y} \Omega \omega \sin \theta-\rho I_{x y} q_{y} \omega^{2} \cos \theta\right] W_{y}} \\
+\left[\kappa_{x} G A k_{x}-q_{x}\left(E I_{y} k_{x}^{2}+\kappa_{x} G A-\rho I_{y} \omega^{2}\right)\right] W_{x}=0 \\
\rho I_{x y} q_{x} \omega^{2} W_{x}-\left[\kappa_{y} G A k_{y}-q_{y}\left(E I_{x} k_{y}^{2}+\kappa_{y} G A-\rho I_{x} \omega^{2}\right)\right] W_{y} \cos \theta=0 .
\end{gathered}
$$

For the spinning beam with arbitrary cross-section, it is apparent that $I_{x}, I_{y}$ and $I_{x y}$ change with $\Omega t$ (or just $t$ when $\Omega$ is constant).

(ii) The $\theta$-factor for the helicity of waves

For a circular beam, the orientation of the cross-section relative to the inertia coordinate system is no longer distinguishable. Thus, $I_{x}=I_{y}=I$ and $I_{x y}=0$. The value zero for $I_{x y}$ leads to the simplification of (2.6) to

$$
2 I \rho q \Omega \omega \cos \theta=0 .
$$

This implies $\theta= \pm(\pi / 2)$ as the only two possible roots for a non-zero $\Omega$. Equation (2.9) gives the same result. It has already been mentioned but will be shown later that the two-valued $\theta$, characterizing the helicity of the waves, arises from the gyroscopic effect, and is thus referred to as the gyroscopic-phase $\theta$-factor.

Table 1 gives the summary of the helicity of the waves. In the table, the sign of $(( \pm \omega) \Omega / \theta)$ is indicative of whether a bending wave in the beam has clockwise (rev-C) or anticlockwise (rev-A) helicity. The frequency $\omega$ is taken as positive. The speed $\Omega$ is positive also taking clockwise spinning of beam as the reference.

It will be shown later that neither the sign of the exponent of $\mathrm{e}^{\mathrm{i}(\omega t \pm k z)}$ or $\mathrm{e}^{-\mathrm{i}(\omega t \pm k z)}$, nor the sign of the $\theta$-factor alone can be used to indicate the helicity of the progressive wave. There is a pattern of signs of $(( \pm \omega) \Omega / \theta)$, as summarized in table 1 , that indicates the helicity of the waves in the beam. It can be seen that, the rev-C waves are associated with $(( \pm \omega) \Omega / \theta)<0$ and the rev-A waves are associated with $(( \pm \omega) \Omega / \theta)>0$.

\section{(iii) Helicity and chirality of the travelling waves}

Geometrically, due to a wave passing through a beam section, the centre of the cross-section has a translated position

$$
\boldsymbol{w}=w_{x} \boldsymbol{e}_{x}+w_{y} \boldsymbol{e}_{y}
$$

where $\boldsymbol{e}_{x}$ and $\boldsymbol{e}_{y}$ are unit vectors in the fixed $x$ - and $y$-axes. For a symmetrical beam, from equations (2.7) and (2.8), it can be shown that $W_{x}=W_{y}=W_{\mathrm{o}}$. Thus, expression (2.5) for the four-component wave function is simplified to

$$
s=\left\{\begin{array}{l}
w_{x} \\
\phi_{y} \\
w_{y} \\
\phi_{x}
\end{array}\right\}=W_{\mathrm{o}}\left\{\begin{array}{c}
1 \\
\pm \mathrm{i} q \\
\mathrm{e}^{\mathrm{i} \theta} \\
\pm \mathrm{ie}^{\mathrm{i} \theta} q
\end{array}\right\} \mathrm{e}^{\mathrm{i}(\omega t \pm k z)}
$$


Table 1. Clockwise (rev-C) and anticlockwise (rev-A) waves-summary of the helicity

\begin{tabular}{|c|c|c|c|}
\hline \multirow[b]{2}{*}{ wavefunction } & \multirow[b]{2}{*}{ spin } & \multicolumn{2}{|l|}{$\Omega>0$} \\
\hline & & $\theta=\pi / 2$ & $\theta=-\pi / 2$ \\
\hline $\begin{array}{l}\mathrm{e}^{\mathrm{i}(\omega t \pm k z)}(+\omega) \\
\mathrm{e}^{-\mathrm{i}(\omega t \pm k z)}(-\omega)\end{array}$ & & $\begin{array}{l}\text { rev-A sign }((+\omega) \Omega / \theta)=(+) \\
\text { rev-C sign }((-\omega) \Omega / \theta)=(-)\end{array}$ & $\begin{array}{l}\text { rev-C sign }((+\omega) \Omega / \theta)=(-) \\
\text { rev-A sign }((-\omega) \Omega / \theta)=(+)\end{array}$ \\
\hline
\end{tabular}

where $q=k-\left(\rho \omega^{2} / \kappa G k\right)$ as defined by Huang (1961). Given $k$ and $\omega, q$ is fixed, and once the amplitude of the centroidal displacement of the cross-section from the equilibrium position is known, the amplitude of the angular orientation of the cross-section is fixed. The centroidal displacement is written as

$$
\boldsymbol{w}=W_{\mathrm{o}} \boldsymbol{e} \angle \varphi,
$$

where $W_{\mathrm{o}}=\sqrt{w_{x}^{2}+w_{y}^{2}}$ is the length of the centroidal displacement vector relative to a point of the cross-section intersecting the fixed $z$-axis. The displacement direction is denoted by the angle $\phi$ that can be regarded as the angle of polarization of the wave. Thus,

The bending angle is

$$
\varphi=\arctan \frac{w_{y}}{w_{x}} .
$$

$$
\boldsymbol{\phi}=\phi_{x} \boldsymbol{e}_{x}+\phi_{y} \boldsymbol{e}_{y}=q W_{\mathrm{o}} \mathrm{e} \angle \varphi_{\phi}
$$

For the FIS wave, substituting expressions in (2.1) or (2.12) into (2.14) and applying Euler's formula yield

$$
\begin{aligned}
\varphi & =\arctan \frac{W_{y} \mathrm{e}^{\mathrm{i}\left(\omega t-k_{y} z\right)} \mathrm{e}^{\mathrm{i} \theta}}{W_{x} \mathrm{e}^{\mathrm{i}\left(\omega t-k_{x} z\right)}} \\
& =\arctan \frac{\left[\cos \left(\omega t-k_{y} z+\theta\right)+\mathrm{i} \sin \left(\omega t-k_{y} z+\theta\right)\right]}{\left[\cos \left(\omega t-k_{x} z\right)+\mathrm{i} \sin \left(\omega t-k_{x} z\right)\right]} .
\end{aligned}
$$

The ratio of the sine imaginary parts or that of the cosine real parts can be used to obtain the following results. However, choosing to use the real parts for a circular beam, equation (2.16) becomes

$$
\begin{gathered}
\varphi=\arctan \left(\frac{\cos (\omega t-k z) \cos \theta-\sin (\omega t-k z) \sin \theta}{\cos (\omega t-k z)}\right) . \\
\text { For } \theta=\frac{\pi}{2}, \quad \varphi=\arctan \left[-\frac{\sin (\omega t-k z)}{\cos (\omega t-k z)}\right] . \\
\text { For } \theta=-\frac{\pi}{2}, \quad \varphi=\arctan \frac{\sin (\omega t-k z)}{\cos (\omega t-k z)} .
\end{gathered}
$$

Thus, when

$$
\frac{\Omega \omega}{\theta}>0, \quad \varphi=-\omega t+k z
$$


and when

$$
\frac{\Omega \omega}{\theta}<0, \quad \varphi=\omega t-k z .
$$

Equation (2.20) represents that the displacement vector $\boldsymbol{w}$ has its polarization revolving in the anticlockwise direction, while (2.21) shows that the polarization revolving in the clockwise direction. At any instant $t$, the anticlockwise wave of (2.20) has the tip of the vector $\boldsymbol{w}$ tracing a right-handed helix (RH-helix) in the $z$-axis, $\varphi=-\omega t+k z$. Similarly, the clockwise wave of $(2.21)$ has the tip of $\boldsymbol{w}$ tracing a LH-helix, $\varphi=\omega t-k z$.

For the BIS wave, $s \sim \mathrm{e}^{\mathrm{i}(\omega t+k z)}$ one may replace equation $(2.17)$ by

$$
\varphi=\arctan \left(\frac{\cos (\omega t+k z) \cos \theta-\sin (\omega t+k z) \sin \theta}{\cos (\omega t+k z)}\right) .
$$

Thus, when

$$
\frac{\Omega \omega}{\theta}>0, \quad \varphi=-\omega t-k z
$$

and when

$$
\frac{\Omega \omega}{\theta}<0, \quad \varphi=\omega t+k z
$$

The anticlockwise wave of (2.23) is a LH-helix while the clockwise wave of (2.24) is a RH-helix. It is remembered that both topological structures correspond to the FIS wave.

(iv) The gyroscopic precession of the spinning beam elements as the waves travel

As shown previously, $W_{x}=W_{y}=W_{\mathrm{o}}$. Normalizing we take $W_{\mathrm{o}}=1$. Each point of the helix revolves at the wave angular frequency $\omega$, tracing a circular orbit of unit length. At each point of the centroidal axis, the cross-section has a bending angular rotation $\boldsymbol{\phi}=q \boldsymbol{e} \angle \varphi_{\phi}$ given by (2.15), being the angle of inclination of the spin axis of each cross-sectional element of the beam with respect to an axis parallel to the $z$-axis, as shown in figure $1 a$ or $b$. Thus, the revolving motion of the travelling wave manifests as a precession of every beam element at the wave frequency $\omega$. The direction of precession depends on the sign of $(\omega \Omega / \theta)$, anticlockwise when it is positive or clockwise when it is negative as shown in table 1.

\section{(d) Frequency splitting due to gyroscopic effect}

It is noted from equation (2.3) that the system operator $\boldsymbol{D}$ represented by (2.2) is skew-symmetric. The $4 \times 4$ matrix is partitioned into four $2 \times 2$ submatrix blocks. Extracting the two off-diagonal blocks for a circular beam we have

$$
\left[\begin{array}{cc}
0 & 0 \\
0 & 2 \Omega \rho I \partial_{t}
\end{array}\right] \text { and }\left[\begin{array}{cc}
0 & 0 \\
0 & -2 \Omega \rho I \partial_{t}
\end{array}\right]
$$


showing the difference in the minus sign. After $\boldsymbol{D}$ operates on the fourcomponent wavefunction of (2.12), we have a new matrix for $\boldsymbol{D}$ written as

$$
\left[\begin{array}{cccc}
-\rho A \omega^{2}+\kappa G A k^{2} & \pm \mathrm{i} \kappa G A k & 0 & 0 \\
\mp \mathrm{i} \kappa G A k & -\rho I \omega^{2}+E I k^{2} & 0 & -2 \mathrm{i} \Omega \omega \rho I \\
& +\kappa G A & & \\
0 & 0 & -\rho A \omega^{2}+\kappa G A k^{2} & \pm \mathrm{i} \kappa G A k \\
0 & 2 \mathrm{i} \Omega \omega \rho I & \mp \mathrm{i} \kappa G A k & -\rho I \omega^{2}+E I k^{2} \\
& & & +\kappa G A
\end{array}\right]
$$

Equation (2.3) may then be divided into two. The upper blocks give

$$
\left[\begin{array}{cc}
-\rho A \omega^{2}+\kappa G A k^{2} & \pm \mathrm{i} \kappa G A k \\
\mp \mathrm{i} \kappa G A k & -\rho I \omega^{2}+E I k^{2}+\kappa G A-2 \mathrm{i} \Omega \omega \rho I \mathrm{e}^{ \pm \mathrm{i}(\pi / 2)}
\end{array}\right]\left\{\begin{array}{c}
1 \\
\pm \mathrm{i} q
\end{array}\right\}=\left\{\begin{array}{l}
0 \\
0
\end{array}\right\}
$$

and the lower blocks yield

$$
\left[\begin{array}{cc}
-\rho A \omega^{2}+\kappa G A k^{2} & \pm \mathrm{i} \kappa G A k \\
\mp \mathrm{i} \kappa G A k & -\rho I \omega^{2}+E I k^{2}+\kappa G A+2 \mathrm{i} \Omega \omega \rho I \mathrm{e}^{\mp \mathrm{i}(\pi / 2)}
\end{array}\right]\left\{\begin{array}{c}
1 \\
\pm \mathrm{i} q
\end{array}\right\}=\left\{\begin{array}{l}
0 \\
0
\end{array}\right\} .
$$

The two equations are exactly similar, and thus the frequency equation is simply written as

$$
\left|\begin{array}{cc}
-\rho A \omega^{2}+\kappa G A k^{2} & \pm \mathrm{i} \kappa G A k \\
\mp \mathrm{i} \kappa G A k & -\rho I \omega^{2}+E I k^{2}+\kappa G A \pm 2 \Omega \omega \rho I
\end{array}\right|=0 .
$$

The sign of the diagonal terms $( \pm i \kappa G A k)$ is related to the sign of the FIS or BIS wave expression. However, this does not matter for calculating the dispersion characteristics, thus

$$
\left|\begin{array}{cc}
-\rho A \omega^{2}+\kappa G A k^{2} & \mathrm{i} \kappa G A k \\
-\mathrm{i} \kappa G A k & -\rho I \omega^{2}+E I k^{2}+\kappa G A \pm 2 \Omega \omega \rho I
\end{array}\right|=0 .
$$

When the spinning speed is zero, $\Omega=0$, this equation corresponds to the dispersion relation (2.6) derived by Chan et al. (2002) who predicted dispersion of two types of travelling waves admissible for the non-spinning Timoshenko beam, the $\mathrm{s}_{\mathrm{a}}$-wave and the $\mathrm{s}_{\mathrm{b}}$-wave. The two types of wave solutions can be considered individually. In this context, however, we do not need to do so. Their propagative characteristics will be affected by the gyroscopic effect in the same way depending on the term $\pm 2 \Omega \omega \rho I$. The 
Table 2. The changes of frequencies due to gyroscopic effect

\begin{tabular}{|c|c|c|c|c|c|}
\hline \multirow{2}{*}{\multicolumn{2}{|c|}{ speed $\left(\right.$ rev s $\left.^{-1}\right)$}} & \multicolumn{4}{|l|}{ frequency, $\omega$} \\
\hline & & $\begin{array}{l}k=2.7 \mathrm{rad} \mathrm{m}^{-1} \\
(\mathrm{kHz})\end{array}$ & $\begin{array}{l}k=6.1 \mathrm{rad} \mathrm{m}{ }^{-1} \\
(\mathrm{kHz})\end{array}$ & $\begin{array}{l}k=9.2 \mathrm{rad} \mathrm{m}^{-1} \\
(\mathrm{kHz})\end{array}$ & $\begin{array}{l}k=15 \mathrm{rad} \mathrm{m} \\
(\mathrm{kHz})\end{array}$ \\
\hline \multirow{3}{*}{$\begin{array}{l}0 \\
2000\end{array}$} & & 10 & 11.3 & 12.6 & 15.8 \\
\hline & $\omega_{1} \downarrow$ & 8.2 & 9.5 & 11 & 14.2 \\
\hline & $\omega_{2} \uparrow$ & 12.2 & 13.2 & 14.5 & 18.7 \\
\hline
\end{tabular}

determinant equation of (2.30) yields a dispersion relation written as

$$
k^{4}-k^{2} \omega^{2}\left(\frac{1}{c_{\mathrm{o}}^{2}}+\frac{1}{c_{2}^{\prime 2}}\right)-\frac{\omega^{2}}{c_{\mathrm{o}}^{2} r_{g}^{2}}+\frac{\omega^{4}}{c_{\mathrm{o}}^{2} c_{2}^{\prime 2}} \mp \frac{2|\Omega \omega|}{c_{\mathrm{o}}^{2}}\left(k^{2}-\frac{\omega^{2}}{c_{2}^{\prime 2}}\right)=0,
$$

where $c_{\mathrm{o}}=\sqrt{\frac{E}{\rho}}, c_{2}^{\prime}=\sqrt{\frac{\kappa G}{\rho}}$ and $r_{\mathrm{g}}=\sqrt{\frac{I}{A}}$. The positive sign is related to $\theta=\pi / 2$ or $(\Omega \omega / \theta)>0$. Thus for the anticlockwise waves, the frequency is lower than the original frequency without the beam spinning. The negative sign of the term $2 \Omega \omega \rho I$ is related to $\theta=-\pi / 2$ or $(\Omega \omega / \theta)<0$. Thus, for the clockwise waves, the frequency is higher than the frequency when the beam is not spinning. Table 2 shows the changes of the frequencies following splitting due to the gyroscopic effect for the beam spinning at $2000 \mathrm{rev} \mathrm{s}^{-1}$ compared with the frequencies for a non-spinning beam, based on equal wavenumber.

\section{(i) Splitting of a plane-polarized wave}

To explain the frequency-splitting wave phenomenon, first consider a nonspinning beam with rotational symmetry, in which any plane containing the $z$-axis has the freedom to admit a plane-polarized wave. For example, if one excites vibration in one plane at a frequency $\omega$, say in the $x z$-plane, the bending wave propagates along $z$-axis in that plane and will not induce wave motion in the orthogonal plane. A non-spinning beam with symmetry admits plane-polarized waves only.

The process of frequency splitting is described as follows. Suppose there is a wave in the $x z$-plane expressed as

$$
\left\{\begin{array}{l}
w_{x} \\
\phi_{y}
\end{array}\right\}=\left\{\begin{array}{c}
1 \\
\pm \mathrm{i} q
\end{array}\right\} \mathrm{e}^{\mathrm{i}(\omega t \pm k z)} .
$$

The gyroscopic effect will induce wave motion in the $y z$-plane, which in turn couples back to the $x z$-plane. Once generated, the interaction is mutual and would lead to an identical wave in the $y z$-plane, given a phase shift, expressed as

$$
\left\{\begin{array}{c}
w_{y} \\
\phi_{x}
\end{array}\right\}=\mathrm{e}^{\mathrm{i} \theta}\left\{\begin{array}{c}
1 \\
\pm \mathrm{i} q
\end{array}\right\} \mathrm{e}^{\mathrm{i}(\omega t \pm k z)}
$$

$\theta$ being either $\pi / 2$ or $-\pi / 2$. The two-valued gyroscopic $\theta$-factor correspond to a rev-A (anticlockwise) or rev-C (clockwise) FIS wave as

$$
\left\{\begin{array}{l}
w_{x} \\
\phi_{y}
\end{array}\right\}=\left\{\begin{array}{c}
1 \\
-\mathrm{i} q
\end{array}\right\} \mathrm{e}^{\mathrm{i}(\omega t-k z)} \text { and }\left\{\begin{array}{l}
w_{y} \\
\phi_{x}
\end{array}\right\}=\left\{\begin{array}{l}
\mathrm{i} \\
q
\end{array}\right\} \mathrm{e}^{\mathrm{i}(\omega t-k z)}
$$


or

$$
\left\{\begin{array}{c}
w_{x} \\
\phi_{y}
\end{array}\right\}=\left\{\begin{array}{c}
1 \\
-\mathrm{i} q
\end{array}\right\} \mathrm{e}^{\mathrm{i}(\omega t-k z)} \text { and }\left\{\begin{array}{l}
w_{y} \\
\phi_{x}
\end{array}\right\}=\left\{\begin{array}{c}
-\mathrm{i} \\
-q
\end{array}\right\} \mathrm{e}^{\mathrm{i}(\omega t-k z)} .
$$

Thus, the gyroscopic coupling leads to the splitting of a plane wave into two waves with opposite helicity and chirality (figure 2).

(ii) Four-component vector representation of the travelling wave solution

For the FIS wave, there are two forms of structural representation, (2.32) and (2.33). They may be combined and expressed as

$$
\left\{\begin{array}{l}
w_{x} \\
\phi_{y} \\
w_{y} \\
\phi_{x}
\end{array}\right\}_{\theta=\pi / 2}=\left\{\begin{array}{c}
1 \\
-\mathrm{i} q \\
\mathrm{i} \\
q
\end{array}\right\} \mathrm{e}^{\mathrm{i}\left(\omega_{1} t-k_{1} z\right)} \text { and }\left\{\begin{array}{c}
w_{x} \\
\phi_{y} \\
w_{y} \\
\phi_{x}
\end{array}\right\}_{\theta=-\pi / 2}=\left\{\begin{array}{c}
1 \\
-\mathrm{i} q \\
-\mathrm{i} \\
-q
\end{array}\right\} \mathrm{e}^{\mathrm{i}\left(\omega_{2} t-k_{2} z\right)}
$$

Similarly, for the BIS wave, the expressions are

$$
\left\{\begin{array}{l}
w_{x} \\
\phi_{y} \\
w_{y} \\
\phi_{x}
\end{array}\right\}_{\theta=\pi / 2}=\left\{\begin{array}{c}
1 \\
\mathrm{i} q \\
\mathrm{i} \\
-q
\end{array}\right\} \mathrm{e}^{\mathrm{i}\left(\omega_{1} t+k_{1} z\right)} \text { and }\left\{\begin{array}{c}
w_{x} \\
\phi_{y} \\
w_{y} \\
\phi_{x}
\end{array}\right\}_{\theta=-\pi / 2}=\left\{\begin{array}{c}
1 \\
\mathrm{i} q \\
-\mathrm{i} \\
q
\end{array}\right\} \mathrm{e}^{\mathrm{i}\left(\omega_{2} t+k_{2} z\right)}
$$

The four vector wavefunctions are four-component vectors, representing the wave entities, implying that the four components in each of these fourcomponent vectors are inseparable and that the dimension of operator $\boldsymbol{D}$ with respect to the four-component vector functions is irreducible. The advantage of this is that a four-component vector wavefunction describes completely all the physical properties of the bending wave it represents, as summarized in table 3 . One further point of significance is that the solutions (2.34) and (2.35) fall into two separated regimes, one belonging to the set of clockwise waves and the other to the set of anticlockwise waves, depending on the sign of $(\Omega \omega / \theta)$ as shown in the table. The problem of interpreting negative-frequency can be avoided by using the two-valued coupling $\theta$-factor for the representation of the helicity. In this table, the 'gyro shifts' column indicates frequency changes of the waves being induced by the gyroscopic effect.

If the four-component vector is to reduce to a lower dimension as $\Omega \rightarrow 0$, it must become a two-component vector because the four-component wave arising from the gyroscopic coupling is associated with frequency splitting, $\omega \rightarrow \omega_{1} \downarrow$ and to $\omega_{2} \uparrow$ as shown in table 2 and figure 2, arrow down indicating frequency decreasing to approach $\omega_{1}$ and arrow up for frequency increasing to approach to $\omega_{2}$. It is interesting to note that, while a change of the two-component wavefunction to a pair of four-component wavefunctions involves 'frequency splitting', conversely the reduction of two relevant four-component vectors into one single two-component vector naturally involves 'merging of a pair of modes' as $\omega_{1} \uparrow \rightarrow \omega$ (increasing to approach $\omega$ ) and $\omega_{2} \downarrow \rightarrow \omega$ (decreasing to approach $\omega$ ). 
Table 3. Properties of bending waves represented by four-component vector wavefunctions

\begin{tabular}{|c|c|c|c|c|c|}
\hline \multirow{2}{*}{$\begin{array}{l}\text { wave type } \\
\text { (equation ref.) }\end{array}$} & \multirow{2}{*}{$\begin{array}{l}\text { four-component } \\
\text { wavefunction }\end{array}$} & \multirow{2}{*}{$\begin{array}{l}(\Omega \omega / \theta) \\
\Omega \omega>0\end{array}$} & \multirow{2}{*}{$\begin{array}{l}\text { polarization, } \varphi(t, z) \\
\text { (equation ref.) }\end{array}$} & \multicolumn{2}{|l|}{ gyro shifts } \\
\hline & & & & chirality $k \mapsto$ & helicity $\omega \mapsto$ \\
\hline \multirow[t]{2}{*}{ FIS $(2.34)$} & $\left\{\begin{array}{c}1 \\
-\mathrm{i} q \\
\mathrm{i} \\
q\end{array}\right\} \mathrm{e}^{\mathrm{i}\left(\omega_{1} t-k_{1} z\right)}$ & $(+)$ & $\varphi=\omega_{1} t+k_{1} z(2.20)$ & RH-helix $k_{1}$ & rev-A $\omega_{1} \downarrow$ \\
\hline & $\left\{\begin{array}{c}1 \\
-\mathrm{i} q \\
-\mathrm{i} \\
-q\end{array}\right\} \mathrm{e}^{\mathrm{i}\left(\omega_{2} t-k_{2} z\right)}$ & $(-)$ & $\varphi=\omega_{2} t-k_{2} z(2.21)$ & LH-helix $k_{2}$ & rev-C $\omega_{2} \uparrow$ \\
\hline \multirow[t]{2}{*}{ BIS (2.35) } & $\left\{\begin{array}{c}1 \\
\mathrm{i} q \\
\mathrm{i} \\
-q\end{array}\right\} \mathrm{e}^{\mathrm{i}\left(\omega_{1} t+k_{1} z\right)}$ & $(+)$ & $\varphi=\omega_{1} t-k_{1} z(2.23)$ & LH-helix $k_{1}$ & rev-A $\omega_{1} \downarrow$ \\
\hline & $\left\{\begin{array}{c}1 \\
\mathrm{i} q \\
-\mathrm{i} \\
q\end{array}\right\} \mathrm{e}^{\mathrm{i}\left(\omega_{2} t+k_{2} z\right)}$ & $(-)$ & $\varphi=\omega_{2} t+k_{2} z(2.24)$ & RH-helix $k_{2}$ & $\mathrm{rev}-\mathrm{C} \omega_{2} \uparrow$ \\
\hline
\end{tabular}

The gyroscopic-coupling effect plays a role in the coupling of the waves in the planes normal to each other. The coupling can be enhanced by the shear deformation. These two factors, $\theta$ and $q$, are both included in the representation of the wavefunction in the form of a four-component vector. Since the two effects give rise to the helicity and chirality of the waves, the exponential expressions $\mathrm{e}^{\mathrm{i}(\omega t-k z)}$ and $\mathrm{e}^{\mathrm{i}(\omega t+k z)}$ alone are not sufficient to represent the properties, as can be seen below.

(iii) Euler's formula and its relation to finding the helical properties

By applying Euler's formula, $A \mathrm{e}^{\mathrm{i}(\omega t-k z)}$ can be expressed as $A \cos (\omega t-k z)+$ $\mathrm{i} A \sin (\omega t-k z)$. Normally one usually takes the real part only as the solution, possibly with an arbitrary phase. However, for a four-component system, the real and imaginary parts of the complex Euler expression are equally important. For instance, from the first equation of (2.34) the translation vector is written as

$$
\begin{gathered}
\left\{\begin{array}{c}
w_{x} \\
w_{y}
\end{array}\right\}_{\theta=\pi / 2}=A\left\{\begin{array}{l}
1 \\
\mathrm{i}
\end{array}\right\} \cos \left(\omega_{1} t-k_{1} z\right)+\mathrm{i} A\left\{\begin{array}{l}
1 \\
\mathrm{i}
\end{array}\right\} \sin \left(\omega_{1} t-k_{1} z\right), \\
\therefore\left\{\begin{array}{l}
w_{x} \\
w_{y}
\end{array}\right\}=A\left\{\begin{array}{c}
\cos \left(\omega_{1} t-k_{1} z\right) \\
-\sin \left(\omega_{1} t-k_{1} z\right)
\end{array}\right\}+\mathrm{i} A\left\{\begin{array}{l}
\sin \left(\omega_{1} t-k_{1} z\right) \\
\cos \left(\omega_{1} t-k_{1} z\right)
\end{array}\right\} .
\end{gathered}
$$


Multiplying a vector by $\mathrm{i}=\mathrm{e}^{\mathrm{i}(\pi / 2)}$ is in effect an operation to rotate the vector by $\pi / 2$ in the complex domain. The measurable real part of equation (2.37) has already reflected this operation by transforming the $w_{y}$ component of the second term of the right hand side of (2.36) to the $w_{y}$ component of the first term of (2.37). Plotting the real part of (2.37) in space and time reveals that the centroidal axis of the beam assumes a helix with anticlockwise helicity at frequency $\omega_{1}$. In fact, what are observed are the projections of the complex $w_{x}$ and $w_{y}$ onto the $x y$-plane. It is understood that the product of the rotation of the cross-section and the spin of the beam, is the factor that gives rise to this gyroscopic-coupling induced $\mathrm{e}^{\mathrm{i} \theta}$ transformation. Therefore, Euler's formula is not used on its own but rather together with the four-component vectors to describe the helical properties of the waves. Given the factors $\theta$ and $q$, the vectors of equations (2.34) can be written in real and imaginary parts as

$$
\left\{\begin{array}{l}
w_{x} \\
\phi_{y} \\
w_{y} \\
\phi_{x}
\end{array}\right\}_{\theta=\pi / 2}=\left\{\begin{array}{c}
1 \\
-\mathrm{i} q \\
\mathrm{i} \\
q
\end{array}\right\} \mathrm{e}^{\mathrm{i}\left(\omega_{1} t-k_{1} z\right)}=\left\{\begin{array}{c}
\cos \left(\omega_{1} t-k_{1} z\right)+\mathrm{i} \sin \left(\omega_{1} t-k_{1} z\right) \\
q \sin \left(\omega_{1} t-k_{1} z\right)-\mathrm{i} q \cos \left(\omega_{1} t-k_{1} z\right) \\
-\sin \left(\omega_{1} t-k_{1} z\right)+\mathrm{i} \cos \left(\omega_{1} t-k_{1} z\right) \\
q \cos \left(\omega_{1} t-k_{1} z\right)+\mathrm{i} q \sin \left(\omega_{1} t-k_{1} z\right)
\end{array}\right\},
$$

and

$$
\left\{\begin{array}{l}
w_{x} \\
\phi_{y} \\
w_{y} \\
\phi_{x}
\end{array}\right\}_{\theta=-\pi / 2}=\left\{\begin{array}{c}
1 \\
-\mathrm{i} q \\
-\mathrm{i} \\
-q
\end{array}\right\} \mathrm{e}^{\mathrm{i}\left(\omega_{2} t-k_{2} z\right)}=\left\{\begin{array}{c}
\cos \left(\omega_{2} t-k_{2} z\right)+\mathrm{i} \sin \left(\omega_{2} t-k_{2} z\right) \\
q \sin \left(\omega_{2} t-k_{2} z\right)-\mathrm{i} q \cos \left(\omega_{2} t-k_{2} z\right) \\
\sin \left(\omega_{2} t-k_{2} z\right)-\mathrm{i} \cos \left(\omega_{2} t-k_{2} z\right) \\
-q \cos \left(\omega_{2} t-k_{2} z\right)-\mathrm{i} q \sin \left(\omega_{2} t-k_{2} z\right)
\end{array}\right\} .
$$

The measurable real parts of these expressions are used to plot the vectors of the centroidal displacement and cross-sectional rotation at different time frames, as shown in figure $3 a$. Equation (2.38) represents a rev-A RH-helix FIS wave while figure $3 b$ represents a rev-C LH-helix FIS wave, consistent with the results about the chirality given in table 3 . It can be seen that while the translation vectors trace a righthanded or left-handed helix, the rotation vectors follow the opposite senses, having opposite helicity and chirality. From these diagrams, it can be seen that, as the wave travels in the spinning beam, it is the helically deformed shape of the centroidal axis that travels, appearing like a corkscrew traveling through the beam.

\section{(e) Helical waves in spinning beam and the whirling of shafts}

The whirling of a shaft is a common phenomenon and has been studied in association with modal balancing and vibrations of flexible rotors (Ginsberg 1995; Bishop \& Parkinson 1968; Morton 1985). It is usually referred to as an excited mode of shaft vibration due to unbalanced forces. If anticlockwise forcing is present and synchronizes with the anticlockwise revolving mode, backward whirl occurs.

In the present context, a revolving mode, either anticlockwise or clockwise, is regarded as the forced revolving mode at one of the natural frequencies of the 
spinning beam, comprising four excited traveling wave entities each being a fourcomponent wavefunction expressed as one of the equations (2.34) and (2.35). These four traveling waves are a FIS $\mathrm{s}_{\mathrm{a}}$, BIS $\mathrm{s}_{\mathrm{a}}$, FIS $\mathrm{s}_{\mathrm{b}}$ and BIS $\mathrm{s}_{\mathrm{b}}$ waves, each revolving at the same frequency and helicity but the FIS and BIS waves are in opposite chirality. The combination of a FIS $s_{a}$ and a BIS $s_{a}$ is a revolving $s_{a}$ standing wave, and the combination of a FIS $\mathrm{s}_{\mathrm{b}}$ and a BIS $\mathrm{s}_{\mathrm{b}}$ wave is a revolving $\mathrm{s}_{\mathrm{b}}$ standing wave. It can be seen that the whirling shape is described as the superposition of the revolving $\mathrm{s}_{\mathrm{a}}$ and $\mathrm{s}_{\mathrm{b}}$ standing waves of the same helicity.

For the four wave entities, the anticlockwise whirling frequency is $\Omega=\omega_{1}$ and the amplitudes are represented as

$$
\begin{aligned}
& \mathbf{S}_{1 \mathrm{aF}}=W_{1 \mathrm{a}}\left\{\begin{array}{llll}
1 & -\mathrm{i} q_{1 \mathrm{a}} & \mathrm{i} & q_{1 \mathrm{a}}
\end{array}\right\}^{\mathrm{T}} \text { and } \\
& \mathbf{S}_{1 \mathrm{bF}}=W_{1 \mathrm{~b}}\left\{\begin{array}{llll}
1 & -\mathrm{i} q_{1 \mathrm{~b}} & \mathrm{i} & q_{1 \mathrm{~b}}
\end{array}\right\}^{\mathrm{T}} \text { for RH-helix, }
\end{aligned}
$$

and

$$
\begin{aligned}
& \mathbf{S}_{1 \mathrm{aB}}=W_{1 \mathrm{a}}\left\{\begin{array}{llll}
1 & \mathrm{i} q_{1 \mathrm{a}} & \mathrm{i} & -q_{1 \mathrm{a}}
\end{array}\right\}^{\mathrm{T}} \text { and } \\
& \mathbf{S}_{1 \mathrm{bB}}=W_{1 \mathrm{~b}}\left\{\begin{array}{llll}
1 & \mathrm{i} q_{1 \mathrm{~b}} & \mathrm{i} & -q_{1 \mathrm{~b}}
\end{array}\right\}^{\mathrm{T}} \text { for LH-helix. }
\end{aligned}
$$

Similarly, the clockwise whirling frequency is $\Omega=\omega_{2}$ and the amplitudes are represented as

and

$$
\begin{aligned}
& \mathbf{S}_{2 \mathrm{aF}}=W_{2 \mathrm{a}}\left\{\begin{array}{llll}
1 & -\mathrm{i} q_{2 \mathrm{a}} & -\mathrm{i} & -q_{2 \mathrm{a}}
\end{array}\right\}^{\mathrm{T}} \text { and } \\
& \mathbf{S}_{2 \mathrm{bF}}=W_{2 \mathrm{~b}}\left\{\begin{array}{llll}
1 & -\mathrm{i} q_{2 \mathrm{~b}} & -\mathrm{i} & -q_{2 \mathrm{~b}}
\end{array}\right\}^{\mathrm{T}} \text { for LH-helix, }
\end{aligned}
$$

$$
\begin{aligned}
& \mathbf{S}_{2 \mathrm{aB}}=W_{2 \mathrm{a}}\left\{\begin{array}{llll}
1 & \mathrm{i} q_{2 \mathrm{a}} & -\mathrm{i} & q_{2 \mathrm{a}}
\end{array}\right\}^{\mathrm{T}} \text { and } \\
& \mathbf{S}_{2 \mathrm{bB}}=W_{2 \mathrm{~b}}\left\{\begin{array}{llll}
1 & \mathrm{i} q_{2 \mathrm{~b}} & -\mathrm{i} & q_{2 \mathrm{~b}}
\end{array}\right\}^{\mathrm{T}} \text { for RH-helix. }
\end{aligned}
$$

Given the relative phases $\delta_{n \mathrm{a}}$ and $\delta_{n \mathrm{~b}}, n=1$ for rev-A or $n=2$ for rev-C wave, the standing waves are expressed as

$$
\begin{aligned}
& \mathbf{s}_{n \mathrm{a}}=\mathbf{S}_{n \mathrm{aF}} \mathrm{e}^{\mathrm{i}\left(\omega_{n \mathrm{a}} t-k_{n \mathrm{a}} z\right)}+\mathbf{S}_{n \mathrm{aB}} \mathrm{e}^{\mathrm{i}\left(\omega_{n \mathrm{a}} t+k_{n a} z\right)} \angle \delta_{n \mathrm{a}} \text { and } \\
& \mathbf{s}_{n \mathrm{~b}}=\mathbf{S}_{n \mathrm{bF}} \mathrm{e}^{\mathrm{i}\left(\omega_{n \mathrm{~b}} t-k_{n \mathrm{~b}} z\right)}+\mathbf{S}_{n \mathrm{bB}} \mathrm{e}^{\mathrm{i}\left(\omega_{n \mathrm{~b}} t+k_{n \mathrm{~b}} z\right)} \angle \delta_{n \mathrm{~b}} .
\end{aligned}
$$

The rev-A RH-helix FIS wave (2.40) superpose with the rev-A LH-helix BIS wave (2.41) to form a rev-A superposed standing $\mathrm{s}_{\mathrm{a}}$ and $\mathrm{s}_{\mathrm{b}}$ wave, similarly for the rev-C FIS (2.42) and BIS (2.43) waves. The superposed standing waves are the normal modes that revolve either anticlockwise or clockwise written, respectively, as

$$
\mathbf{s}_{1}=2\left[W_{1 \mathrm{a}}\left\{\begin{array}{c}
\cos \left(k_{1 \mathrm{a}} z-\delta_{1 \mathrm{a}}\right) \\
-q_{1 \mathrm{a}} \sin \left(k_{1 \mathrm{a}} z-\delta_{1 \mathrm{a}}\right) \\
\mathrm{i} \cos \left(k_{1 \mathrm{a}} z-\delta_{1 \mathrm{a}}\right) \\
-\mathrm{i} q_{1 \mathrm{a}} \sin \left(k_{1 \mathrm{a}} z-\delta_{1 \mathrm{a}}\right)
\end{array}\right\}+W_{1 \mathrm{~b}}\left\{\begin{array}{c}
\cos \left(k_{1 \mathrm{~b}} z-\delta_{1 \mathrm{~b}}\right) \\
-q_{1 \mathrm{~b}} \sin \left(k_{1 \mathrm{~b}} z-\delta_{1 \mathrm{~b}}\right) \\
\mathrm{i} \cos \left(k_{1 \mathrm{~b}} z-\delta_{1 \mathrm{~b}}\right) \\
-\mathrm{i} q_{1 \mathrm{~b}} \sin \left(k_{1 \mathrm{~b}} z-\delta_{1 \mathrm{~b}}\right)
\end{array}\right\}\right] \mathrm{e}^{\mathrm{i} \omega_{1} t}
$$




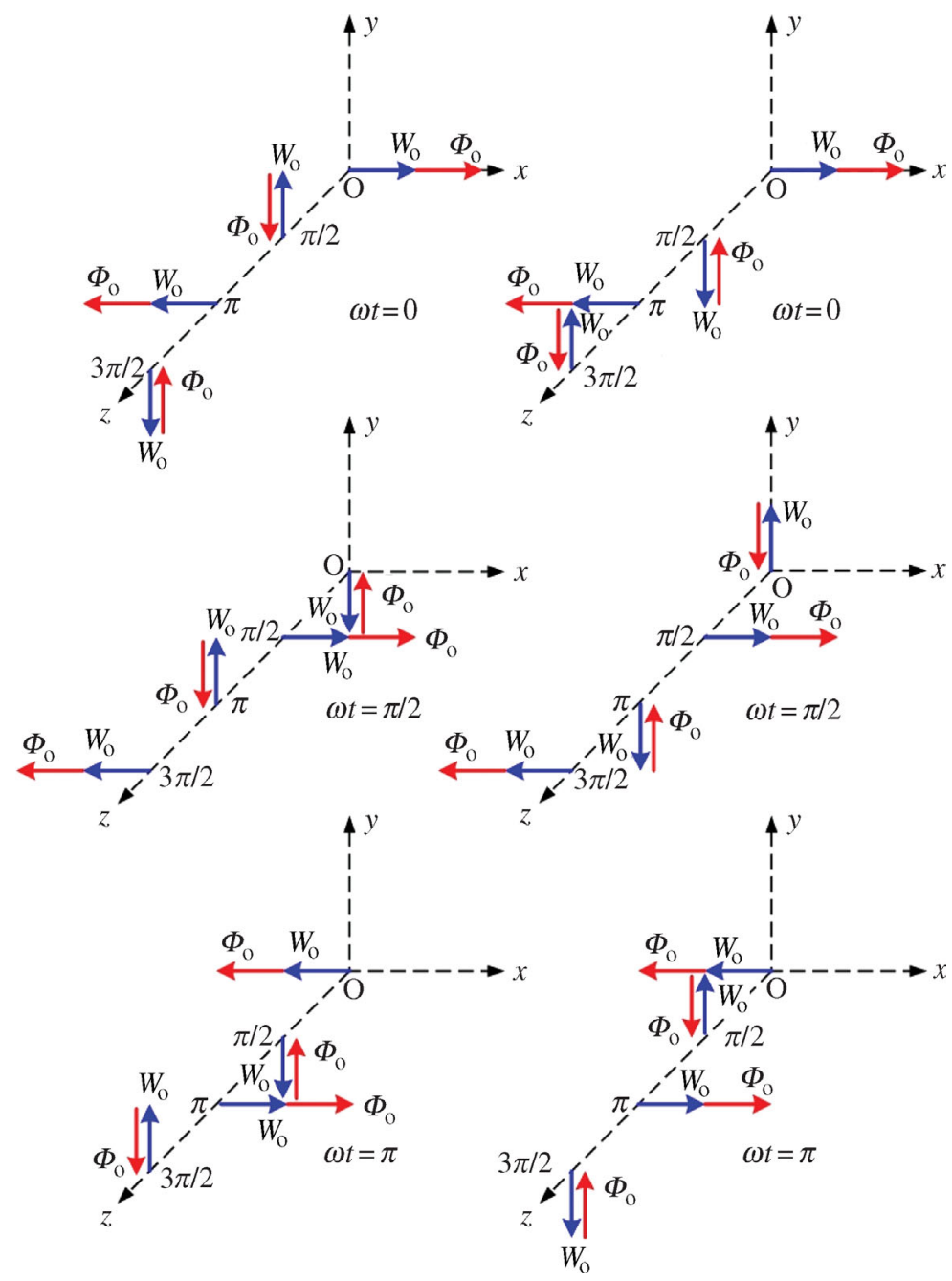

Figure 3. Displacement vectors of the centroids of the beam and bending rotation vectors of the cross-section at different time frames for the FIS waves. (a) For equation (2.37) rev-A RH-helix structure. (b) For equation (2.38) rev-C LH-helix structure.

or

$$
\mathbf{s}_{2}=2\left[W_{2 \mathrm{a}}\left\{\begin{array}{c}
\cos \left(k_{2 \mathrm{a}} z-\delta_{2 \mathrm{a}}\right) \\
-q_{2 \mathrm{a}} \sin \left(k_{2 \mathrm{a}} z-\delta_{2 \mathrm{a}}\right) \\
-\mathrm{i} \cos \left(k_{2 \mathrm{a}} z-\delta_{2 \mathrm{a}}\right) \\
\mathrm{i} q_{2 \mathrm{a}} \sin \left(k_{2 \mathrm{a}} z-\delta_{2 \mathrm{a}}\right)
\end{array}\right\}+W_{2 \mathrm{~b}}\left\{\begin{array}{c}
\cos \left(k_{2 \mathrm{~b}} z-\delta_{2 \mathrm{~b}}\right) \\
-q_{2 \mathrm{~b}} \sin \left(k_{2 \mathrm{~b}} z-\delta_{2 \mathrm{~b}}\right) \\
-\mathrm{i} \cos \left(k_{2 \mathrm{~b}} z-\delta_{2 \mathrm{~b}}\right) \\
\mathrm{i} q_{2 \mathrm{~b}} \sin \left(k_{2 \mathrm{~b}} z-\delta_{2 \mathrm{~b}}\right)
\end{array}\right\}\right] \mathrm{e}^{\mathrm{i} \omega_{2} t}
$$

In these equations, $W_{1 \mathrm{a}}, W_{2 \mathrm{a}}, W_{1 \mathrm{~b}}, W_{2 \mathrm{~b}}, \delta_{1 \mathrm{a}}, \delta_{2 \mathrm{a}}, \delta_{1 \mathrm{~b}}$ and $\delta_{2 \mathrm{~b}}$ are arbitrary constants representing the amplitudes and relative phases, to be determined by the boundary conditions that the relevant normal mode has to satisfy. The rev-A or rev-C normal mode is referred to as backward or forward shaft whirling.

In (2.38) and (2.39) for progressive waves, it can be seen that time and space variables cannot be separated, while in (2.45) and (2.46) for standing waves, the 
variables are separable with the spatial parts as a four-component vector wavefunction. This describes a superposed sinusoidal wave lying in a plane, revolving at a frequency either anticlockwise at $\omega_{1}$ as in the time function $\mathrm{e}^{\mathrm{i} \omega_{1} t}$ or clockwise $\omega_{2}$ in $\mathrm{e}^{\mathrm{i} \omega_{2} t}$.

\section{(f) Interpretation of vibration in relation to the helical wave features}

The solution given by Chan et al. (2002) shows that there are two wavenumbers $\left(k_{\mathrm{a}}\right.$ and $\left.k_{\mathrm{b}}\right)$ and two frequencies $\left(\omega_{\mathrm{a}}\right.$ and $\left.\omega_{\mathrm{b}}\right)$ of different types, the $\mathrm{s}_{\mathrm{a}}$-wave and the $\mathrm{s}_{\mathrm{b}}$-wave types. The waves are different physical entities with different phase speeds and group behaviours. The wavenumbers, $k_{\mathrm{a}}$ and $k_{\mathrm{b}}$, are the roots of the following fourth order algebraic equation,

$$
k^{4}-k^{2} \omega^{2}\left(\frac{1}{c_{\mathrm{O}}^{2}}+\frac{1}{c_{2}^{\prime 2}}\right)-\frac{\omega^{2}}{c_{\mathrm{O}}^{2} r_{g}^{2}}+\frac{\omega^{4}}{c_{\mathrm{O}}^{2} c_{2}^{\prime 2}}=0 .
$$

Given the relation $\left(\omega_{\mathrm{a}, \mathrm{b}} / k_{\mathrm{a}, \mathrm{b}}\right)=c_{\mathrm{Pa}, \mathrm{Pb}}$, the wave speeds can be expressed as functions of either the frequency or the wavenumber to describe the dispersion behaviour of the respective waves. In this case, the two $\mathrm{s}_{\mathrm{a}}$ and $\mathrm{s}_{\mathrm{b}}$ standing waves in the finite beam are the two individual standing waves vibrating at a synchronous frequency in superposition to satisfy the boundary conditions. Each standing wave $\left(\mathrm{s}_{\mathrm{a}}\right.$ or $\left.\mathrm{s}_{\mathrm{b}}\right)$ consists of a FIS and a BIS progressive waves, polarized in the same plane with no revolving motion. Thus the helicity and chirality properties do not seem to be relevant to the vibration study.

However, should a slight perturbation occur to produce a non-zero spinning speed, for each of the progressive waves, the gyroscopic splitting process described in figure 2 for the FIS wave occurs, the same for the BIS wave though not shown in the figure. Thus, a pair of rev-A waves at the lower frequency $\omega_{1}$ appears, from which one finds that the FIS wave has a RH-helicity and the BIS one has a LHhelicity. Similarly, a pair of rev-C waves at the higher frequency $\omega_{2}$ arises, in which the FIS wave has a LH-helicity and the BIS one has a RH-helicity. The rev-A and rev-C travelling waves would have the same frequencies $\omega_{1}$ and $\omega_{2}$ but opposite helicity when the perturbation of spinning is virtually zero. They superpose one on the other to form the two revolving normal modes, which in turn superpose to form a non-revolving normal mode that satisfies the boundary conditions. Thus, the helical features are hidden in the case of beam vibration.

\section{(g) The local energy equality property and the dispersion characteristics}

Jeffcott (1919) showed that when the shaft is whirling, its spinning speed equals its natural frequency

$$
\Omega=\omega=\sqrt{\frac{K_{\mathrm{s}}}{m_{\mathrm{R}}}},
$$

where $m_{\mathrm{R}}$ is a mass concentrated at the middle of the span and $K_{\mathrm{s}}$ is the stiffness provided by the massless shaft. The planar shape of the whirling shaft is regarded as a revolving normal mode in the context of this paper. The mass $m_{\mathrm{R}}$ travels on a circular orbit with a constant kinetic energy equal to the shaft potential energy,

$$
T=\frac{1}{2} m_{\mathrm{R}}\left(\mathrm{a}_{\mathrm{o}}\right)^{2} \omega^{2} \text { and } \quad V=\frac{1}{2} K_{\mathrm{S}}\left(\mathrm{a}_{\mathrm{o}}\right)^{2} .
$$


Thus, the Jeffcott single-mass system always has the energy equality property, $T=V$.

As far as the helical wave in the spinning beam considered above is concerned, each of the cross-sectional elements can be considered as a Jeffcott mass displaced by a constant radius, the centroids of the entire series of beam elements tracing the helix as previously mentioned. The difference here is that each orbiting beam element has a rotational motion as it circles the orbit and a potential energy because of elastic shear and bending deformations of the element itself. The result is that local kinetic and potential energies at every position along the helix are equal. It is necessary to show under what condition each of the elements of the beam possesses equal kinetic and potential energies when a wave travels along the beam, and to compare the predicted dispersion relationship with one that is established elsewhere.

\section{(i) The energy expressions}

With the shape of the Timoshenko beam as a centroidal helix, it is assumed that the internal bending moments and shear stresses are balanced by the centrifugal force per unit length $\left(m W_{\mathrm{o}} \omega^{2}\right)$ due to the revolving motion. Let the helix have pitch $\lambda=(2 \pi / k)$, angular frequency $\omega$, and radius $W_{\mathrm{o}}$. The tangential velocity of each of the masses is $W_{\mathrm{o}} \omega$. For simplicity, it is assumed that there is a slight spinning only, i.e. $\Omega \rightarrow 0$, and thus $\omega_{1} \rightarrow \omega$ and $\omega_{2} \rightarrow \omega$. The local $K E$ (kinetic energy) density is

$$
K E=\frac{1}{2} m W_{\mathrm{o}}^{2} \omega^{2}+\frac{1}{2} \rho I\left(\omega q W_{\mathrm{o}}\right)^{2} .
$$

The local $P E$ (potential energy) density is

$$
\begin{aligned}
P E & =\frac{1}{2}\left[E I\left(\partial_{z} \phi\right) \times\left(\partial_{z} \phi\right)^{*}\right]+\frac{1}{2}\left[\kappa G A\left(\partial_{z} w-\phi\right) \times\left(\partial_{z} w-\phi\right)^{*}\right] \\
& =\frac{1}{2} E I(k q)^{2} W_{\mathrm{o}}^{2}+\frac{1}{2} \kappa G A(k-q)^{2} W_{\mathrm{o}}^{2},
\end{aligned}
$$

where $*$ denotes the complex conjugate. From the above, it can be seen that $K E$ and $P E$ are constant, implying that the beam element behaves like a Jeffcott mass at the revolving frequency $\omega$.

\section{(ii) The role of centrifugal force on a revolving helix}

In this context, the physical significance of $q$ is now related to the 'centrifugal force' that keeps the helix in shape. This can be seen from the definition of $q$ as

$$
\frac{q}{k}=\frac{\tan \Phi_{\mathrm{o}}}{k W_{\mathrm{o}}}=1-\frac{m \omega^{2} W_{\mathrm{o}}}{k^{2} W_{\mathrm{o}} \kappa A G},
$$

for small $\Phi_{\mathrm{o}}$. In the case of an Euler-Bernoulli beam, the relation $\tan \Phi_{\mathrm{o}}=k W_{\mathrm{o}}$ is simply a geometric expression for a helix. If there is shear deformation, the second part of (2.52) represents the force ratio between the centrifugal force and the shear force per unit length of the helical structure. 
(iii) The condition for the local equality of $K E$ and $P E$

Equating equations (2.50) and (2.51) gives the condition for the local energy equality property of the deformed helix kept in balance by the centrifugal force

$$
\frac{1}{2} m \omega^{2}+\frac{1}{2} \rho I(\omega q)^{2}=\frac{1}{2} E I(k q)^{2}+\frac{1}{2} \kappa G A(k-q)^{2} .
$$

Eliminating $q,(2.53)$ becomes

$$
\left(k^{2}-\frac{m \omega^{2}}{\kappa G A}\right)\left[k^{4}-\omega^{2} k^{2}\left(\frac{m}{E A}+\frac{m}{\kappa G A}\right)+\left(\frac{m^{2} \omega^{4}}{\kappa G A E A}-\frac{m \omega^{2}}{\kappa G A I}\right)\right]=0 .
$$

Expressing the equation in terms of wave properties radius of gyration $r_{g}$, sound speed $c_{\mathrm{o}}$ and shear wave speed $c_{2}^{\prime},(2.54)$ is rewritten as

$$
k^{2}-\frac{\omega^{2}}{c_{2}^{\prime 2}}=0 \Rightarrow c_{2}^{\prime}=\frac{\omega}{k},
$$

or

$$
k^{4}-\omega^{2} k^{2}\left(\frac{1}{c_{o}^{2}}+\frac{1}{c_{2}^{\prime 2}}\right)+\left(\frac{\omega^{4}}{c_{o}^{2} c_{2}^{\prime 2}}-\frac{\omega^{2}}{c_{o}^{2} r_{g}^{2}}\right)=0 .
$$

Equation (2.55) suggests that there is a shear wave in action. Equation (2.56) is similar to the dispersion relation for a non-spinning Timoshenko beam. This shows that the model of a helical structure revolving at a speed $\omega$ kept in balance by the centrifugal force is equivalent to a helical wave passing through a beam with a dispersion characteristic described by equation (2.56).

\section{(h) Comparing with the results of Main, Elmore and Heald}

The implications of comparing equation (2.56) with the results of Main (1984), and Elmore \& Heald (1985), are two-fold. One is that the revolving gyroscopic helical structure as depicted in the previous sections, with local energy distribution satisfying equality (2.56), is consistent with the general feature of a travelling wave. That is, like other types of waves, it has the local energy equality property and has an associated definite dispersion relationship (2.56). The other and more interesting implication is that the equality (2.53) is subtly different from the result for plane-polarized travelling waves on a string (Main 1984; Elmore \& Heald 1985) or on a slender Euler-Bernoulli beam (Elmore \& Heald 1985). The subtlety lies in the difference in the structure of the travelling waves. In the present paper, we interpret a travelling wave as one that has chirality and helicity, even when the spinning speed is closing to zero. Table 4 shows the energy equality features of different types of waves. From this table, the only way to reach a complete similarity of wave behaviour between them is to eliminate the difference mentioned above. The obvious alternative is to consider each type of travelling waves, shown in the table, as the superposition of a pair of oppositely revolving waves with chirality and helicity. The conclusion, therefore, is that all waves introduced in the table have chirality and helicity properties. 
Table 4. Features of the energy density equality relation for different types of waves

\begin{tabular}{|c|c|c|c|}
\hline $\begin{array}{l}\text { traveling wave type } \\
\text { and media }\end{array}$ & $\begin{array}{l}\text { energy density equality } \\
\text { relation } K E=P E\end{array}$ & $\begin{array}{l}K E \text { and } P E \text { in } \\
\quad \text { relation to } t\end{array}$ & $\begin{array}{l}\text { equations, equal } \\
\text { energy relations } \\
\text { and reference }\end{array}$ \\
\hline $\begin{array}{l}\text { transverse, on string, } \\
\text { non-dispersive, } \\
\text { sinusoidal }\end{array}$ & $\begin{array}{c}\frac{1}{2} \rho c^{2} k^{2} W_{\mathrm{o}}^{2} \sin ^{2}(k z-\omega t)= \\
\frac{1}{2} T k^{2} W_{\mathrm{o}}^{2} \sin ^{2}(k z-\omega t)\end{array}$ & $\begin{array}{l}K E \text { and } P E \\
\text { change with } t \\
\text { ave. equal }\end{array}$ & $\begin{array}{c}\rho \omega^{2}=T k^{2}(\text { Elmore } \\
\& \text { Heald 1985; } \\
\text { Main 1984) }\end{array}$ \\
\hline $\begin{array}{l}\text { longitudinal on thin } \\
\text { rod, non-dispersive, } \\
\text { sinusoidal }\end{array}$ & $\begin{array}{r}\frac{1}{2} \rho A \omega^{2} W_{\mathrm{o}}^{2} \sin ^{2}(k z-\omega t)= \\
\frac{1}{2} A E k^{2} W_{\mathrm{o}}^{2} \sin ^{2}(k z-\omega t)\end{array}$ & $\begin{array}{l}K E \text { and } P E \\
\text { change with } t \\
\text { ave. equal }\end{array}$ & $\begin{array}{l}E k^{2}=\rho \omega^{2}(\text { Elmore \& } \\
\quad \text { Heald 1985) }\end{array}$ \\
\hline $\begin{array}{l}\text { bending on thin rod, } \\
\text { dispersive, sinusoidal }\end{array}$ & $\begin{array}{r}\frac{1}{2} \rho A \omega^{2} W_{\mathrm{o}}^{2} \sin ^{2}(k z-\omega t)= \\
\frac{1}{2} E I k^{4} W_{\mathrm{o}}^{2} \sin ^{2}(k z-\omega t)\end{array}$ & $\begin{array}{l}K E \text { and } P E \\
\text { change with } t \\
\text { ave. equal }\end{array}$ & $\begin{array}{c}E l k^{4}=\rho A \omega^{2}(\text { Elmore } \\
\& \text { Heald 1985) }\end{array}$ \\
\hline $\begin{array}{l}\text { revolving wave on } \\
\text { Timoshenko beam, } \\
\text { dispersive, helical }\end{array}$ & equation (2.53) & $\begin{array}{c}K E \text { and } P E \\
\text { constant }\end{array}$ & equation (2.54) \\
\hline
\end{tabular}

\section{Conclusion}

The literature on vibration and wave phenomena associated with a spinning beam has been reviewed, showing the interest in gyroscopic effect over the last two decades. Thus, our study has concentrated on finding the cause of the frequency-splitting phenomenon that takes place in a spinning Timoshenko beam. In effect, the present study has helped shed light on the relationship between the wave motion in a spinning beam and the whirling of a shaft. The following conclusions can be drawn.

(i) The progressive bending waves in a spinning beam with circular crosssection are circularly polarized. They can be physically described as two distinct sets of revolving helices, each of which is traced by the centroidal axis of the spinning beam. One set consists of clockwise-revolving helices and the other set consists of anticlockwise-revolving helices. A two-valued phase $\mathrm{e}^{\mathrm{i} \theta}$ is used to represent the helicity of the wave, which arises when the gyroscopic effect causes the progressive waves in orthogonal planes to undergo mutual interaction and replaces a plane-polarized wave in beam with no spinning by two helical waves with opposite helicity. Each of the helices has a chirality property, either right-handedness or left-handedness. For each of these helical waves, the beam elements behave as a gyroscopic disc with clockwise or anticlockwise precession at the wave frequency.

(ii) The frequency-splitting phenomenon is accompanied by two circularly polarized progressive waves, of opposite helicity, with higher and lower wavenumbers respectively, in accordance with their actual dispersion circumstances and is consistent with the gyroscopic influence. If the beam has a finite length, in addition to the gyroscopic effect, the dispersion circumstances are uniquely determined by the boundary conditions.

(iii) In a spinning Timoshenko beam of finite length, all standing waves are revolving as plane-polarized waves with a helicity property. A normal mode 
is therefore a superposition of a revolving $\mathrm{s}_{\mathrm{a}}$-standing and a revolving $\mathrm{s}_{\mathrm{b}}$ standing wave, both with the same helicity. Thus, the frequency-splitting process generates one clockwise revolving normal mode at a higher frequency and an anticlockwise revolving normal mode at a lower frequency. These two unequal normal mode frequencies merge to an equal one and the two revolving normal modes, retaining their own intrinsic but opposite helicity, superpose to form a plane-polarized normal mode vibrating up and down. From this perspective, one may consider that a normal mode of vibration in a stationary beam consists of two revolving normal modes of vibration of two hidden but opposite helicities. If a normal mode is excited by an unbalanced force in a spinning shaft system, the shaft is said to be in synchronous whirling.

(iv) In order to show the progressive wave properties of the helical structure traced by the centroidal axis of the beam, a reverse approach can be used: first specify the dimensions of the structural helix; second calculate its internal elastic energies per unit length; third calculate its kinetic energy given the revolving speed. Then, the local energy equality result for progressive wave is applied to obtain the dispersive relation of the bending wave in the beam. From the consistency of the prediction with the results of Chan et al. (2002), we conclude that the helical structure depicted behaves as a traveling wave.

The first author acknowledges the grants CERG PolyU 5119/98E, PolyU5148/00E, PolyU5163/02E, and ITF grant UIT/003 with thanks. He is grateful to Professor Sir Gabriel Harn and Professor Christopher Calladine for their valuable discussions on the structures of DNA helices. Thanks are also due to Mr. Cai Ting and Mr. Huang Jun for their valuable help in preparing the final version of the figures.

\section{References}

Argento, A. \& Scott, R. A. 1995 Elastic wave propagation in a Timoshenko beam spinning about its longitudinal axis. Wave Motion 21, 67-74. (doi:10.1016/0165-2125(94)00045-7.)

Bauer, H. F. 1980 Vibration of a rotating uniform beam, Part 1: orientation in the axis of rotation. J. Sound Vib. 72, 177-189. (doi:10.1016/0022-460X(80)90651-3.)

Bishop, R. E. D. 1959 The vibration of rotating shafts. J. Mech. Eng. Sci. 1, 50.

Bishop, R. E. D. \& Parkinson, A. G. 1968 Vibration and balancing of flexible shafts. Appl. Phys. Rev. 21, 439-451.

Chan, K. T., Wang, X. Q., So, R. M. C. \& Reid, S. R. 2002 Superposed standing waves in a Timoshenko beam. Proc. R. Soc. A 458, 83-108. (doi:10.1098/rspa.2001.0855.)

Elmore, W. C. \& Heald, M. A. 1969 Physics of waves, pp. 31-38, 96-97, 117-119. New York: McGraw-Hill.

Ginsberg, J. H. 1995 Advanced engineering dynamicsi. 2nd edn, pp. 439-440. Cambridge University Press.

Huang, T. C. 1961 The effect of rotatory inertia and of shear deformation on the frequency and normal mode equations of uniform beams with simple end conditions. ASME J. Appl. Mech. 28, 579-584.

Jeffcott, H. H. 1919 The lateral vibration of loaded shafts in the neighbourhood of a whirling speed-the effect of want of balance. Phil. Mag. 37, 304.

Kang, B. \& Tan, C. A. 1998 Elastic wave motions in an axially strained, infinitely long rotating Timoshenko shaft. J. Sound Vib. 213, 467-482. (doi:10.1006/jsvi.1998.1516.) 
Lee, C. W., Katz, R., Ulsoy, A. G. \& Scott, R. A. 1988 Modal analysis of a distributed parameter rotating shaft. J. Sound Vib. 122, 119-130.

Main, I. G. 1984 Vibrations and waves in physics. 2nd edn, pp. 139-273. Cambridge University Press.

Morton, P. G. 1985 Modal balancing of flexible shaft without trial weights. Proc. I. Mech. E 199, 71-78.

Morton, P. G. \& Johnson, J. H. 1980 The use and interpretation of vibration measurements. Tribol. Int. 5, 225-231. (doi:10.1016/0301-679X(80)90078-X.)

Tan, C. A. \& Kang, B. 1998 Wave reflection and transmission in an axially strained, rotating Timoshenko shaft. J. Sound Vib. 213, 483-510. (doi:10.1006/jsvi.1998.1517.)

Zu, J. W. Z. \& Han, R. P. S. 1992 Natural frequencies and normal modes of a spinning Timoshenko beam with general boundary conditions. ASME J. Appl. Mech. 59, 197-204.

As this paper exceeds the maximum length normally permitted, the authors have agreed to contribute to production costs. 